\title{
COMPARACIÓN DEL RENDIMIENTO DE LOS COMANDOS INSERT, SELECT Y DELETE EN LOS SISTEMAS GESTORES DE BASES DE DATOS ORACLE Y MYSQL ${ }^{1}$
}

\author{
COMPARING THE EFFICIENCY OF THE INSERT, SELECT AND DELETE COMMANDS IN \\ ORACLE AND MYSQL RELATIONAL DATABASE MANAGEMENT SYSTEMS \\ COMPARAÇÃO DO RENDIMENTO DOS COMANDOS INSERT, SELECT E DELETE NOS \\ SISTEMAS GESTORES DE BANCOS DE DADOS ORACLE E MYSQL \\ Johan Alexander Portilla Tovar²; Mireya Bernal Gómez ${ }^{3}$
}

Fecha de recibido: Agosto 06 de 2017 | Fecha de aprobado: Octubre 03 de 2017

\section{Resumen}

Oracle y Mysql son gestores de bases de datos relacionales (con versiones libres) utilizados en el campo informático. Este artículo presenta los resultados de la comparación del rendimiento de los comandos Insert, Select y Delete de estos dos motores de bases de datos. Si bien la sintaxis de codificación de uno y otro gestor es similar, se tiende a pensar erróneamente que ocurre lo mismo con los resultados de ejecución de las sentencias mencionadas. Por esto, con este documento se demuestra cuál de estos dos motores de bases de datos es más eficiente al ejecutar cada uno de los comandos mencionados.

Se implementó un esquema de base de datos y se incluyeron varios registros con el comando Insert, para luego llevar a cabo los procesos con los comandos Select y Delete. Los resultados obtenidos se presentan de forma gráfica, en cuadros comparativos que evidencian qué motor de bases de datos es mejor para cada comando ejecutado con cierta cantidad de información, o, en su defecto, si el resultado obtenido en ambos motores de bases de datos es el mismo.

Palabras clave: Oracle, Mysql, SQL, DML DML, Gestores de Bases de datos relacionales.

1 Artículo de investigación.

2 Estudiante de Tecnología en Informática. Corporación Universitaria Minuto de Dios -UNIMINUTO. Correo electrónico: jportillato@uniminuto.edu.co

3 Docente de Tecnología en Informática. Corporación Universitaria Minuto de Dios -UNIMINUTO. Correo electrónico: mbernal@uniminuto.edu.co 


\section{Abstract}

Oracle and MySQL are open-source relational database management systems used in computing. This article presents the results of a comparison of the efficiency of the Insert, Select and Delete commands when using these two database engines. Although both systems have a similar encoding syntax, people mistakenly think that the same is true for the execution results of the statements. Thus, through this paper we attempt to prove which of them is more efficient when executing the three commands mentioned.

We implemented a database schema and included a number of registers using the Insert command. Then, we did the same with the Select and Delete commands. The results are displayed graphically, in comparative charts showing whether one database engine is better for each command executed with a certain amount of information, or, otherwise, if the results obtained by using both database engines are the same.

Keywords: Oracle, MySQL, SQL, DML, relational database management systems

\section{Resumo}

Oracle e Mysql são gestores de bancos de dados relacionais (com versões livres) utilizados no campo informático. Este artigo apresenta os resultados da comparação do rendimento dos comandos Insert, Select e Delete destes dois motores de bancos de dados. Embora a sintaxe de codificação de um e outro gestor são semelhantes, se tende a pensar de maneira errónea, que ocorre o mesmo com os resultados de execução das sentenças mencionadas. Por isso, com este documento se demonstra qual destes dois motores de bancos de dados é mais eficiente ao executar a cada um dos comandos mencionados.

Implementou-se um esquema de banco de dados e incluíram-se vários registros com o comando Insert, para depois levar a cabo os processos com os comandos Select e Delete. Os resultados obtidos apresentam-se de forma gráfica, em quadros comparativos que evidenciam qué motor de bases de dados é melhor para cada comando executado com certa quantidade de informação, ou, em seu defeito, se o resultado obtido em ambos motores de bancos de dados é o mesmo.

Palavras-chave: Oracle, Mysql, SQL, DML, Relational Database Managers. 
Esta investigación establece una comparación entre el rendimiento de dos motores de bases de datos relacionales (Oracle y Mysql), teniendo en cuenta los comandos Insert, Select y Delete, con el fin de dar a conocer por medio de pruebas computacionales cuál de estos sistemas gestores de bases de datos (SGBDR) es más eficiente a la hora de ejecutar los comandos mencionados, o, en su defecto, determinar si hay igualdad en las respuestas obtenidas. Para ello se realizó un recorrido por diferentes sentencias en Lenguaje Estructurado de Consultas (SQL) que fueron ejecutadas en ambos gestores de bases de datos, para puntualizar qué tan favorable puede ser cada uno; además, se muestran tablas de comparación con los resultados obtenidos en cada proceso de ejecución.

Se escoge a Oracle y Mysql como motores de bases de datos para realizar las pruebas, ya que permiten probar, con cierta cantidad de registros, el tiempo de respuesta que tardan en llevar a cabo la petición realizada (Cardona, Rendón \& Reyes, 2016; García, 2017).

El enfoque que se da a este artículo está dirigido a un entorno educativo, ya que las plataformas y gestores con los que se realizan las pruebas son las que actualmente se usan en este ámbito. Además, puede ser de utilidad para quienes se inicien como desarrolladores en bases de datos, bien en un programa tecnológico, bien en uno profesional o en el área de la informática.

Según Medrano y Torreño (2016), entre los gestores de bases de datos Oracle y Mysql, en lo que respecta a su rendimiento, Mysql tiene mejores resultados en las sentencias de actualización y borrado de datos; pero Oracle es más rápido en la realización de consultas. De acuerdo con Zhingri y Augusto (2016), quienes comparan el rendimiento de Mysql con MongODB, se determina que, en cuanto a seguridad, Mysql es la mejor opción; sin embargo, Mongodb es más veloz. En el estudio de Pilco Guachi (2012) se hace una comparación entre Mysql y Firebird, en el cual se evidencia que Firebird tiene un mejor desempeño en tiempos de respuesta en las consultas, aunque no sea superior en características. En este estudio se ejecutan tres comandos Select, con pocos registros, lo cual no genera sensación de una visualización de resultados completa.

La investigación de Inzunza (2016) realizó un análisis comparativo de tres gestores de bases de datos relacionales (SQL Server 2012, Postgre SQL y Mysql), en donde se determina que Mysql y SQL Server 2012, al tener aspectos cualitativos iguales, presentaron comportamientos similares a la hora de dar respuestas a las consultas realizadas; sin embargo, Postgres SQL presentó mejores resultados sobre estos gestores.

Cuervo (2012), comparando el rendimiento de dos gestores de bases de datos relacionales, Mysql y Firebird, obtuvo como resultado que Mysql es más rápido al ejecutar las transacciones realizadas. No obstante, este artículo aclara que el criterio de resultado de todos los estudios que se hacen depende del proyecto en el que vayan a ser ejecutados, $y$, por lo tanto, no se puede decir si un motor de bases de datos es mejor que otro.

\section{METOdOLOGÍA}

En esta investigación se llevaron a cabo los procedimientos relacionados a continuación:

1. Se instalaron dos motores de bases de datos relacionales: Mysql y Oracle. (En la Tabla 1 se presentan las características del equipo en el que se hizo la instalación).

2. Se ejecutaron las sentencias Select, Insert y Delete con una cantidad de datos que van desde 10000 hasta 50000 registros, con los cuales se lleva a cabo el proceso de comparación.

3. Se tabularon los resultados obtenidos en las comparaciones realizadas y se dedujeron las conclusiones.

Tabla 1. Computador utilizado

\begin{tabular}{|c|c|}
\hline \multicolumn{2}{|c|}{$\begin{array}{c}\text { CARACTERÍSTICAS COMPUTADOR UTILIZADO } \\
\text { PARA REALIZAR PRUEBAS }\end{array}$} \\
\hline Procesador & $\begin{array}{l}\text { AMD Athlon II X4 } 645 \text { HD } \\
\text { Graphics } 3.1 \mathrm{GHz}\end{array}$ \\
\hline Memoria RAM & 4,00 GB DDR3 \\
\hline Disco Duro & HTS SATA Disk Device 1 TB \\
\hline Sistema Operativo & Windows 864 Bits \\
\hline Video & $\begin{array}{l}\text { AMD Radeon HD } 6310 \\
\text { Graphics }\end{array}$ \\
\hline
\end{tabular}

Fuente: elaboración propia.

A continuación, se detallan los procedimientos realizados en la investigación: 


\section{Instalación y manipulación}

Oracle: La herramienta usada es Oracle SQL Developer (versión libre), el manejo de base de datos con el programa ORACLE XE

Mysql: Se utiliza la plataforma de uso libre llamada XAMPP.

A continuación, en la tabla 2 , se evidencian las características de los gestores de bases de datos utilizados.

Tabla 2. Características de los gestores usados

\begin{tabular}{|l|c|c|}
\hline & Oracle & Mysql \\
\hline Versión & $11 \mathrm{~g}$ & 5.6 .16 \\
\hline $\begin{array}{l}\text { Plataforma de } \\
\text { manejo }\end{array}$ & Oracle XE v11.2 & XAMPP v3.2.1 \\
\hline Codificación & $\begin{array}{c}\text { Oracle SQL } \\
\text { Develomper v4.2 }\end{array}$ & $\begin{array}{c}\text { PHPMyAdmin } \\
\text { v4.7.1 }\end{array}$ \\
\hline Servidor & $127.0 .0 .1: 8080$ & $\begin{array}{c}127.0 .0 .1 \text { via } \\
\text { TCP/IP }\end{array}$ \\
\hline
\end{tabular}

Fuente: elaboración propia.

El almacenamiento de cada gestor se muestra a continuación:

Mysql: En el disco duro y memoria Ram (no se requiere una capacidad específica) el computador nativo casero es suficiente, en este caso de 1 тв у 4 Gв, respectivamente (Mysql, 2017).

Oracle: Solo permite $4 \mathrm{~GB}$ de almacenamiento y/o 1 GB de memoria RAM. Al superar este límite, Oracle ofrece otras versiones completas, en las que hay que pagar licencias (Oracle, 2017).

Al usar las versiones libres y no completas de estos dos gestores, lo que implica ciertas limitaciones, se estipula que las pruebas que se hacen aquí son enfocadas a educadores y estudiantes, ya que estos gestores se usan habitualmente para propósitos de enseñanza y aprendizaje. También está enfocado a quienes estén en introducción al campo de las bases de datos.

El proceso para la obtención de datos que se van a evaluar en las tablas es complejo. En este caso, se utiliza un Script de datos de uso libre, cuya autoría es anónima, pero que trae Oracle XE a la hora de su instalación en el usuario predefinido "HR". Este script contiene la creación de las tablas, sus relaciones y la inserción de los datos necesarios, lo cual se puede descargar de:

Mysql: http://bit.ly/2AxAo5g

Oracle: http://bit.ly/2hiiP0o

Los Scripts son de uso libre y contienen las sentencias necesarias que se aplican en las pruebas. En este caso se usan con fines netamente didácticos, y se preserva la protección de datos personales, si así es el caso. Para estas pruebas, en el caso de Oracle XE, no se utiliza el usuario por defecto que trae "SYSTEM" ni tampoco el usuario cuyo esquema es "HR"; más bien, se crea un usuario y se copia el Script en él, para manejar la base de datos de manera autónoma. En el caso de Mysql no se realizan creaciones de usuario, simplemente se copia el Script para crea una base de datos. Al finalizar el proceso de la codificación de los Scripts en sus respectivos gestores, se obtiene el modelo Entidad-Relación de la base de datos, el cual se presenta en la Figura 1:

Figura 1. Modelo Entidad-Relación de la base de datos utilizada

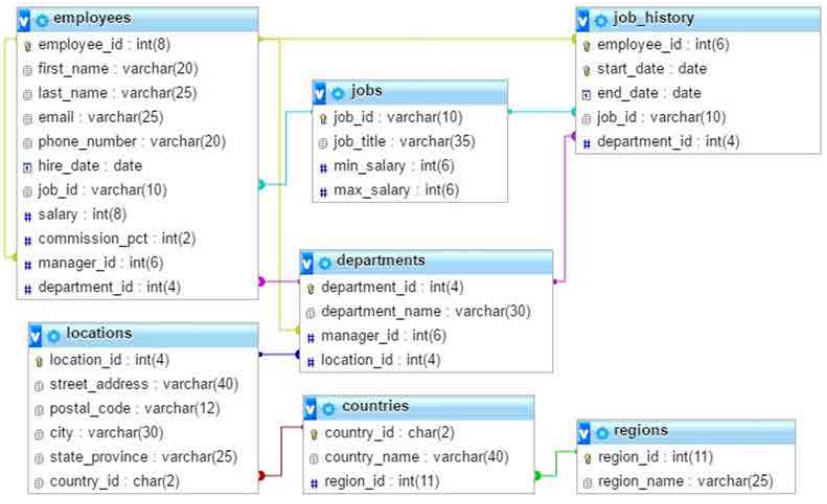

Fuente: elaboración propia.

La carga de datos es similar en ambos gestores; sin embargo, hay ciertas diferencias en su codificación, y por eso cada sentencia que se evalúa aportará, bien para los dos gestores, o para cada uno por aparte, según sus diferencias. En estas sentencias se maneja el lenguaje sQL, el cual es el estándar en los Sistemas de Gestión de Bases de Datos (Torres, López \& Flores, 2016, p. 1). Se cuenta con la ventaja de que los mismos gestores arrojan los tiempos verídicos de ejecución justo después de finalizar cada proceso.

A continuación, se realizará la ejecución de las sentencias a evaluar; se muestra una tabla de resultados obtenidos, un gráfico comparativo y un análisis del proceso. 
La visión global de los comandos a valorar se presenta a continuación (Díez del Valle \& Torreño, 2016, p. 47):

- Inserción de datos

- Consultas sencillas

- Consultas complejas

- Ordenaciones

- Agrupaciones

- Combinaciones entre tablas (Joins) simples

- Combinaciones entre tablas (Joins) múltiples

- Subconsultas simples

- Subconsultas múltiples

- Borrados

\section{Pruebas Insert}

Tipo: Inserción de datos

Descripción: Este comando engloba la creación de los registros necesarios en la base de datos. Se insertan los registros en la tabla EMPLOYEes de manera lógica, teniendo en cuenta sus relaciones en la base de datos.

El en siguiente vínculo puede descargarse del archivo que contiene el Script por ejecutar: http://bit. $\mathrm{ly} / 2 \mathrm{q} 60 \mathrm{sTg}$

Para las pruebas, se insertaron $10000,20000,30000$, 40000 y/o 50000 registros, según corresponda. En la Tabla 3 se muestra la cantidad de registros insertados, los cuales van desde 10000 hasta 50000 , determinando el tiempo de respuesta arrojado por los dos motores de bases de datos en cada una de las iteraciones realizadas (para este caso 8 ).

Tabla 3. Resultados prueba Insert

\begin{tabular}{|r|c|c|c|c|c|c|c|c|c|c|}
\hline Registros $\rightarrow$ & \multicolumn{2}{|c|}{10000} & \multicolumn{2}{c|}{20000} & \multicolumn{2}{c|}{30000} & \multicolumn{2}{c|}{40000} & \multicolumn{2}{c|}{50000} \\
\hline & \multicolumn{2}{|c|}{ Tiempo (seg) } & \multicolumn{2}{|c|}{ Tiempo (seg) } & \multicolumn{2}{c|}{ Tiempo (seg) } & \multicolumn{2}{c|}{ Tiempo (seg) } & \multicolumn{2}{c|}{ Tiempo (seg) } \\
\hline Iterción $\downarrow$ & Oracle & Mysql & Oracle & Mysq) & Oracle & Mysql & Oracle & Mysql & Oracle & Mysql \\
\hline $1 \rightarrow$ & 91,821 & 28,550 & 181,453 & 58,930 & 275,292 & 86,420 & 340,557 & 130,320 & 402,065 & 159,570 \\
\hline $2 \rightarrow$ & 75,307 & 30,980 & 200,088 & 72,090 & 279,462 & 82,590 & 371,165 & 123,270 & 419,304 & 165,700 \\
\hline $3 \rightarrow$ & 68,765 & 27,260 & 175,143 & 62,630 & 256,088 & 79,010 & 355,082 & 137,240 & 439,653 & 160,340 \\
\hline $4 \rightarrow$ & 68,606 & 28,250 & 201,139 & 70,450 & 270,024 & 83,330 & 373,458 & 137,196 & 427,174 & 158,070 \\
\hline $5 \rightarrow$ & 77,859 & 27,090 & 200,932 & 60,270 & 268,992 & 85,090 & 338,670 & 131,656 & 411,553 & 146,590 \\
\hline $6 \rightarrow$ & 83,789 & 29,020 & 191,609 & 59,230 & 282,342 & 80,010 & 349,099 & 129,116 & 398,799 & 143,150 \\
\hline$\rightarrow \rightarrow$ & 81,232 & 28,580 & 204,600 & 54,900 & 270,077 & 88,150 & 365,032 & 140,577 & 423,226 & 142,290 \\
\hline $8 \rightarrow$ & 92,614 & 28,220 & 179,091 & 59,210 & 262,552 & 78,300 & 354,546 & 138,465 & 404,079 & 149,644 \\
\hline Promedio $\rightarrow$ & 79,999 & 28,494 & 191,757 & 62,214 & 270,604 & 82,863 & 355,951 & 133,480 & 415,732 & 153,169 \\
\hline
\end{tabular}

Fuente: elaboración propia.
Figura 2. Resultados prueba Insert 10000

Resultados obtenidos por los dos motores de bases de datos al insertar 10000 registros en 8 iteraciones

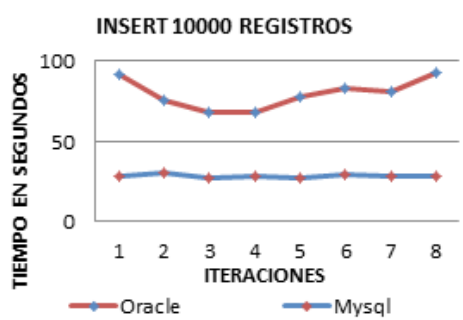

Fuente: elaboración propia.

Figura 3. Resultados prueba Insert 20000 Resultados obtenidos por los dos motores de bases de datos al insertar 20000 registros en 8 iteraciones

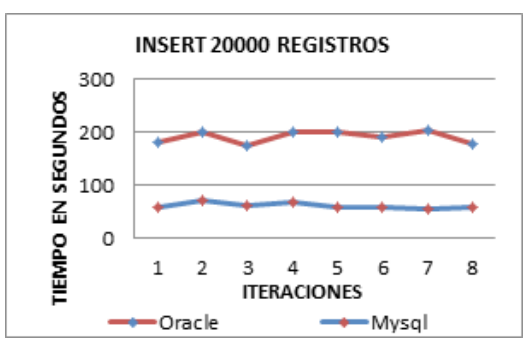

Fuente: elaboración propia.

Figura 4. Resultados prueba Insert 30000

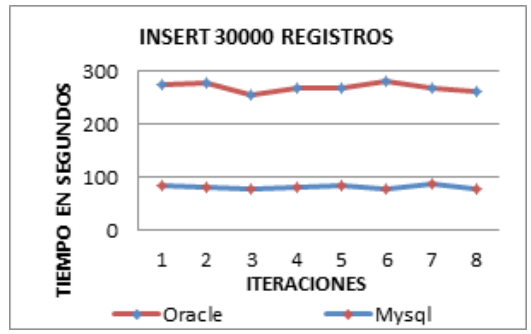

Fuente: elaboración propia.

Figura 5. Resultados prueba Insert 40000

Resultados obtenidos por los dos motores de bases de datos al insertar 40000 registros en 8 iteraciones

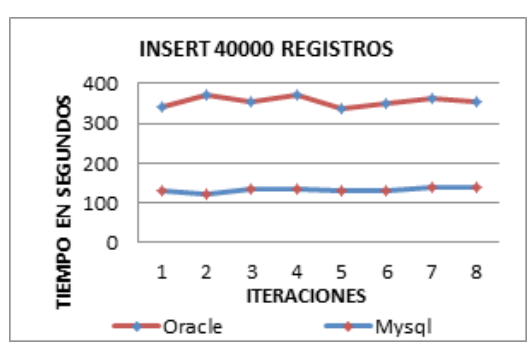

Fuente: elaboración propia. 
Figura 6. Resultados prueba Insert 50000

Resultados obtenidos por los dos motores de bases de datos al insertar 50000 registros en 8 iteraciones

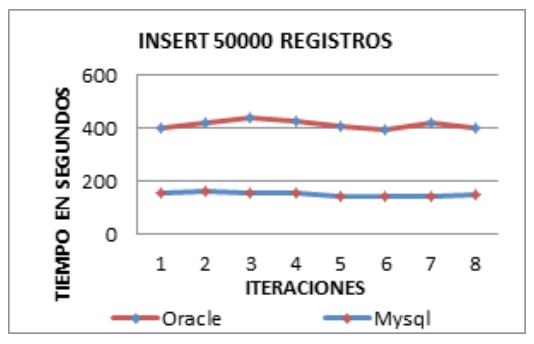

Fuente: elaboración propia.

Al analizar las figuras 2, 3, 4, 5 y 6 se observa que el tiempo de ejecución de la sentencia Insert es demasiado alto en ambos gestores; sin embargo, Mysql logra una mayor eficiencia, ya que obtiene un tiempo casi 3 veces menor. Mysql demoró en promedio 92 segundos, mientras que Oracle unos 262 segundos. Se observa de igual manera que Mysql oscila entre los 27 y 165 segundos al ejecutar la inserción de los datos, mostrando así constancia en cada proceso; en cambio Oracle muestra mucha variación, oscilando entre 68 y 439 segundos. 10000 registros insertados en Mysql demoran aproximadamente 31 segundos; mientras que, en Oracle, unos 84 segundos.

\section{Pruebas Select}

En la Tabla 4 se enlistan los comandos de consulta que se utilizaron para evaluar el rendimiento en ambos motores de bases de datos. De cada sentencia se realizaron 5 iteraciones, con el fin de tomar los tiempos de ejecución y sacar el tiempo promedio (como se muestran en las Tablas 5, 6, 7, 8, 9, 10, 11, 12, 13; y luego en las Figuras 7, 8, 9, 10, 11, 12, $13,14,15,16,17,19,20,21$ se muestra el análisis gráfico que describe de los resultados obtenidos.

Tabla 4. Sentencias Select a evaluar

\begin{tabular}{|r|l|c|}
\hline Grupo No. & Tipo de Agrupación & Consulta(s) \\
\hline 1 & Consulta sencilla & 1,2 \\
\hline 2 & Consulta única & 3 \\
\hline 3 & Condición múltiple & 4,5 \\
\hline 4 & Ordenación & 6 \\
\hline 5 & Agrupación y condiciones de grupo & 7,8 \\
\hline 6 & Combinación entre tablas (JOIN SIMPLE) & 9 \\
\hline 7 & Combinación entre tablas (JOIN MÚLTIPLE) & 10 \\
\hline 8 & Subconsulta simple & 11 \\
\hline 9 & Subconsulta múltiple & 12 \\
\hline
\end{tabular}

Fuente: Tipos de consulta que se llevan a cabo (Díez del Valle \& Torreño, 2016).

\section{Consulta número 1}

Tipo: Consulta sencilla

Descripción: Traer todos los registros de la tabla EMPLOYEES.

Sentencia para los dos gestores: Select * from Employees.

Datos recuperados: 50107

Tabla 5. Resultados de la consulta número 1 en los dos motores de bases de datos

\begin{tabular}{|c|c|c|}
\hline & \multicolumn{2}{|c|}{ Tiempo (seg) } \\
\hline Iteración $\downarrow$ & Oracle & Mysql \\
\hline $1 \rightarrow$ & 5,583 & 1,804 \\
\hline $2 \rightarrow$ & 6,571 & 1,815 \\
\hline $3 \rightarrow$ & 5,385 & 1,808 \\
\hline $4 \rightarrow$ & 5,496 & 1,808 \\
\hline $5 \rightarrow$ & 5,356 & 1,800 \\
\hline Promedio & 5,678 & 1,807 \\
\hline
\end{tabular}

Fuente: elaboración propia.

Figura 7. Resultados de la consulta número 1 obtenidos por los dos motores de bases de datos

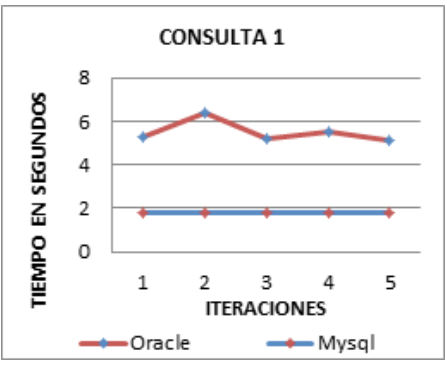

Fuente: elaboración propia.

De acuerdo con la Tabla 5 y la Figura 7, se puede decir que, al ejecutar una sentencia de consulta sencilla, el tiempo de ejecución en Mysql es 3 veces inferior, en promedio, que en Oracle, ya que varía en 1,2 segundos, de acuerdo a las 5 iteraciones realizadas, mientras que Mysql varía en solo 0,01 segundos, mostrando así mayor efectividad en la ejecución de la respuesta.

\section{Consulta número 2}

Tipo: Consulta sencilla

Descripción: Traer el nombre y cargo concatenado de la tabla EMPLOYEes. 
Sentencia para Oracle: Select first_name || 'Es un' I job_id as "cargos" from employees.

Sentencia para Mysql: Select CONCAT (first_name, 'Es un', job_id) as “cargos” from employees

Datos recuperados: 50107

Tabla 6. Promedio de resultados de la consulta número 2 en los dos motores de base de datos

\begin{tabular}{|r|r|r|}
\cline { 2 - 3 } \multicolumn{1}{c|}{} & \multicolumn{2}{c|}{ Tiempo (seg) } \\
\hline Iteración $\downarrow$ & Oracle & Mysql \\
\hline $\mathbf{1} \rightarrow$ & 4,000 & 1,032 \\
\hline $\mathbf{2} \rightarrow$ & 4,304 & 1,026 \\
\hline $\mathbf{3} \rightarrow$ & 4,653 & 1,032 \\
\hline $4 \rightarrow$ & 4,174 & 1,024 \\
\hline $5 \rightarrow$ & 4,553 & 1,038 \\
\hline Promedio & 4,337 & 1,030 \\
\hline
\end{tabular}

Fuente: elaboración propia.

Figura 8. Resultados de la consulta número 2 obtenidos por los dos motores de bases de datos

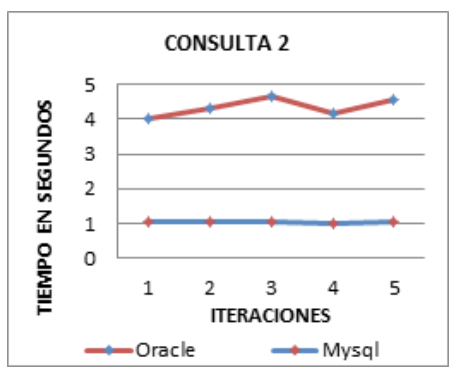

Fuente: elaboración propia.

De acuerdo con la Tabla 6 y la Figura 8 se puede decir que, al ejecutar una sentencia de consulta concatenada sencilla, en Mysql el tiempo de respuesta es 4 veces más rápido, en promedio, que en Oracle. Además, el tiempo varía más en Oracle que en Mysql.

\section{Consulta número 3}

Tipo: Consulta única

Descripción: Traer los campos ID y cargo de la tabla EMPLOYEES Cuyo id sea "10200"

Sentencia para los dos gestores: Select employee id, job_id from employees where employee_id = '10200'
Datos recuperados: 1

Tabla 7. Resultados de la consulta número 3 obtenidos por los dos motores de bases de datos

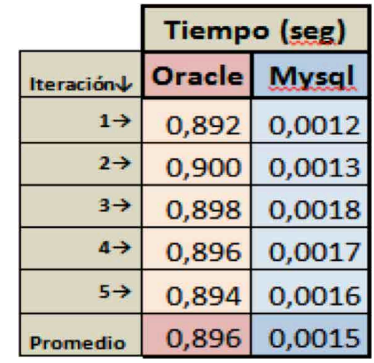

Fuente: elaboración propia.

Figura 9. Resultados de la consulta número 3 obtenidos por los dos motores de bases de datos

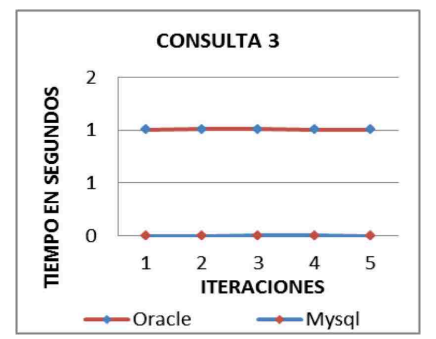

Fuente: elaboración propia.

De acuerdo con la Tabla 7 y Figura 9, se puede decir que, al ejecutar una sentencia de consulta única, los tiempos promedios medidos por ambos gestores se mantienen constantes: Mysql lo hace casi 1 segundo más rápido que Oracle.

\section{Consulta número 4}

Tipo: Condición múltiple

Descripción: Traer los campos nombre, apellido y email de la tabla EMPLOYEEs que comiencen con las letras B, C, D, E, F, G o H, mediante el operador OR.

Sentencia para los dos gestores: select first_name, last_name, email from employees where first_name like ' $\mathrm{B} \%$ ' or first_name like ' $\mathrm{C} \%$ ' or first_name like 'D\%' or first_name like 'E\%' or first_name like 'F\%' or first_name like 'G\%' or first_name like ' $\mathrm{H} \%$ '.

Datos recuperados: 18561 
Tabla 8. Resultados de la consulta número 4 en los dos motores de bases de datos

\begin{tabular}{|r|r|r|}
\cline { 2 - 3 } \multicolumn{1}{c|}{} & \multicolumn{2}{c|}{ Tiempo (seg) } \\
\hline Iteración $\downarrow$ & Oracle & Mysql \\
\hline $1 \rightarrow$ & 1,295 & 1,040 \\
\hline $\mathbf{2} \rightarrow$ & 1,383 & 1,036 \\
\hline $3 \rightarrow$ & 1,197 & 1,042 \\
\hline $4 \rightarrow$ & 1,508 & 1,047 \\
\hline $5 \rightarrow$ & 1,168 & 1,041 \\
\hline Promedio & 1,310 & 1,041 \\
\hline
\end{tabular}

Fuente: elaboración propia.

Figura 10. Resultados obtenidos de la consulta número 4 por los dos motores de bases de datos

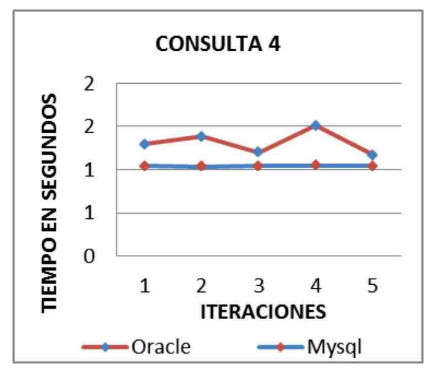

Fuente: elaboración propia.

De acuerdo con la tabla 8 y la Figura 10, que comparan los tiempos de ejecución de una condición múltiple, se puede decir que Oracle presenta una mejor respuesta frente a las consultas anteriores; sin embargo, Mysql logra sobreponerse por un promedio de 0,3 segundos.

\section{Consulta número 5}

Tipo: Condición múltiple

Descripción: Traer los campos nombre y el teléfono de los registros de la tabla EMPLOYEes cuyo departamento sea 50,60, 80, 100 mediante el operador IN.

Sentencia para los dos gestores: Select first_name, phone_number from employees where department id IN (50, 60, 80, 100);

Datos recuperados: 47090
Tabla 9. Resultados de la consulta número 5 en los dos motores de bases de datos

\begin{tabular}{|r|r|r|}
\cline { 2 - 3 } \multicolumn{1}{c|}{} & \multicolumn{2}{c|}{ Tiempo (seg) } \\
\hline Iteración $\downarrow$ & Oracle & Mysql \\
\hline $1 \rightarrow$ & 2,595 & 2,627 \\
\hline $2 \rightarrow$ & 2,683 & 2,636 \\
\hline $3 \rightarrow$ & 2,537 & 2,599 \\
\hline $4 \rightarrow$ & 2,601 & 2,607 \\
\hline $5 \rightarrow$ & 2,565 & 2,611 \\
\hline Promedio & 2,596 & 2,616 \\
\hline
\end{tabular}

Fuente: elaboración propia.

Figura 11. Resultados obtenidos de la consulta número 5 por los dos motores de bases de datos

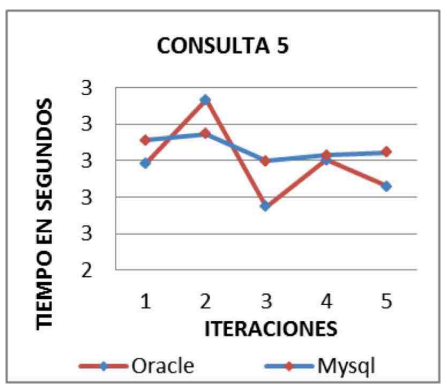

Fuente: elaboración propia.

De acuerdo con la Tabla 9 y la Figura 11, se puede observar que, al ejecutar una sentencia de consulta múltiple, Oracle gana en tiempo promedio por solo 0,02 segundos. Sin embargo, ambos gestores manifiestan mucha variación en sus tiempos de respuesta.

\section{Consulta número 6}

Tipo: Ordenación

Descripción: Traer los registros de la tabla EMPLOYEES cuyo salario esté entre 6000 y 12000 , ordenado por la columna salario. Se utiliza el operador BETWEEN.

Sentencia para los dos gestores: Select * from employees where salary BETWEEN 6000 and 12000 order by salary.

Datos recuperados: 23335 
Tabla 10. Resultados de la consulta número 6 en los dos motores de bases de datos

\begin{tabular}{|r|r|r|}
\cline { 2 - 3 } \multicolumn{1}{c|}{} & \multicolumn{2}{c|}{ Tiempo (seg) } \\
\hline Iteración $\downarrow$ & Oracle & Mysql \\
\hline $\mathbf{1} \rightarrow$ & 4,173 & 7,555 \\
\hline $\mathbf{2} \rightarrow$ & 4,105 & 7,370 \\
\hline $3 \rightarrow$ & 4,115 & 7,725 \\
\hline $4 \rightarrow$ & 4,007 & 7,536 \\
\hline $5 \rightarrow$ & 4,252 & 7,255 \\
\hline Promedio & 4,130 & 7,488 \\
\hline
\end{tabular}

Fuente: elaboración propia.

Figura 12. Resultados obtenidos de la consulta número 6 por los dos motores de bases de datos

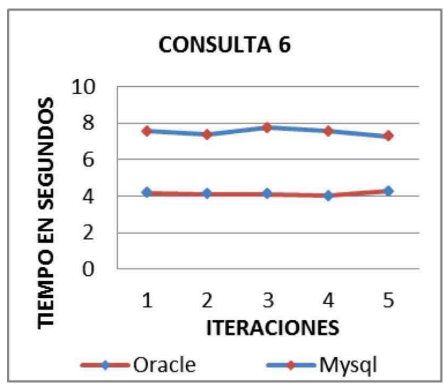

Fuente: elaboración propia.

De acuerdo con la Tabla 10 y la Figura 12, se observa que, al ejecutar una sentencia de ordenación de datos, Oracle tiene un tiempo de respuesta más prolongado que el de Mysql, el cual lo hace casi el doble de rápido. Su diferencia total promedio es de 3,3 segundos. Se muestra constancia en ambos gestores.

\section{Sentencias Delete}

A continuación, en la Tabla 11, se muestra la cantidad de registros, que van de 10000 a 50 000, con respecto a los cuales se determina el tiempo de respuesta de los dos gestores frente a la sentencia Delete. El proceso se lleva a cabo con un total de 8 iteraciones, para una mayor efectividad del estudio.

Tipo: Borrado

Descripción: Borrar los registros de los empleados.

Sentencia para los dos gestores: Delete from employees where employee_id between 207 and *1

$1 \mathrm{El}$ asterisco corresponde al dato donde va a llegar.
Datos borrados: 10 000*, 20 000*, 30 000*, 40 000* y/o $50000 *$.

Tabla 11. Resultados de la prueba Delete

\begin{tabular}{|c|c|c|c|c|c|c|c|c|c|c|}
\hline \multirow{3}{*}{$\begin{array}{l}\text { Registros } \\
\text { Iteración } \downarrow\end{array}$} & \multirow{2}{*}{\multicolumn{2}{|c|}{$\frac{10000}{\text { Tiempo (seg) }}$}} & \multicolumn{2}{|c|}{20000} & \multicolumn{2}{|c|}{30000} & \multicolumn{2}{|c|}{40000} & \multicolumn{2}{|c|}{50000} \\
\hline & & & Tiempc & - (seg) & Tiemp & ० (seg) & Tiempe & $\circ$ (seg) & Tiempc & o (seg) \\
\hline & Oracle & Mysql & Oracle & Mysql & Oracle & Mysql & Oracle & | Mysq| & Oracle & Mysql \\
\hline $1 \rightarrow$ & 4,932 & 1,184 & 6,121 & 0,637 & 8,003 & 0,870 & 10,726 & 0,988 & 12,965 & 1,240 \\
\hline $2 \rightarrow$ & 4,246 & 0,333 & 6,598 & 0,653 & 9,607 & 0,905 & 10,442 & 0,995 & 13,341 & 1,312 \\
\hline $3 \rightarrow$ & 5,355 & 0,305 & 7,355 & 0,644 & 8,432 & 0,853 & 11,002 & 1,003 & $\mid 13,347$ & 1,201 \\
\hline $4 \rightarrow$ & 5,901 & 0,313 & 6,504 & 0,656 & 9,235 & 0,863 & 10,126 & 1,076 & 13,276 & 1,200 \\
\hline $5 \rightarrow$ & 4,724 & 0,334 & 6,209 & 0,636 & 8,234 & 0,899 & 10,234 & 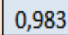 & 12,812 & 1,106 \\
\hline $6 \rightarrow$ & 5,507 & 0,326 & 6,890 & 0,650 & 8,279 & 0,859 & 10,548 & 1,009 & 12,775 & 1,258 \\
\hline$\rightarrow \rightarrow$ & 5,823 & 0,328 & 5,289 & 0,624 & 9,926 & 0,901 & 10,962 & 1,028 & 12,491 & 1,157 \\
\hline $8 \rightarrow$ & 5,021 & 0,322 & 6,095 & 0,635 & 9,301 & 0,852 & 10,803 & 1,004 & 13,035 & 1,250 \\
\hline medio & 5,439 & 0,431 & 6,383 & 0,642 & 8,877 & 0,875 & 10,605 & 1,011 & 13,005 & 1,21 \\
\hline
\end{tabular}

Fuente: Tiempo de respuesta en borrado de datos con cierto número de registros (Almonacid, 2016).

A continuación, se muestra en las Figuras 13, 14, 15,16 y 17 el tiempo de ejecución de borrado de acuerdo a la cantidad de registros.

Figura 13. Resultados prueba Delete 10000

Resultados obtenidos en tiempo por los dos motores de bases de datos al llevar a cabo el proceso de borrar 10000 registros

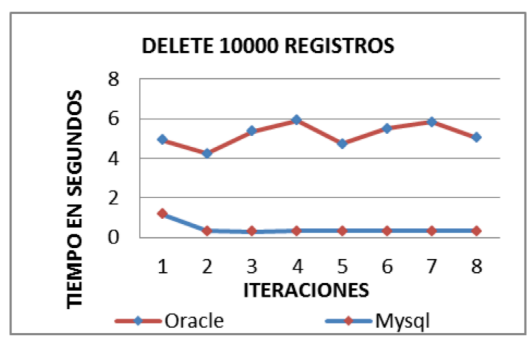

Fuente: elaboración propia.

Figura 14. Resultados prueba Delete 20000 Resultados obtenidos en tiempo por los dos motores de bases de datos al llevar a cabo el proceso de borrar 20000 registros

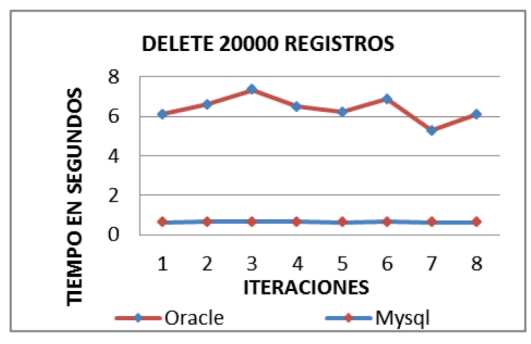

Fuente: elaboración propia. 
Figura 15: Resultados prueba Delete 30000 Resultados obtenidos en tiempo por los dos motores de bases de datos al llevar a cabo el proceso de borrar 30000 registros

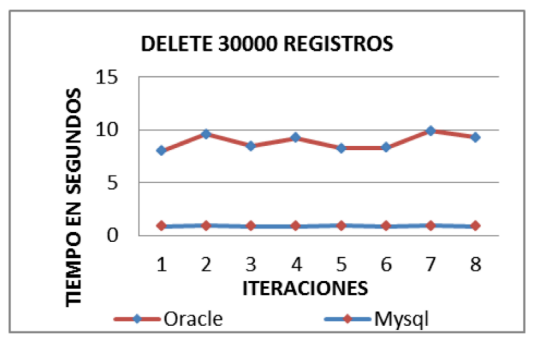

Fuente: elaboración propia.

Figura 16. Resultados prueba Delete 40000 Resultados obtenidos en tiempo por los dos motores de bases de datos al llevar a cabo el proceso de borrar 40000 registros

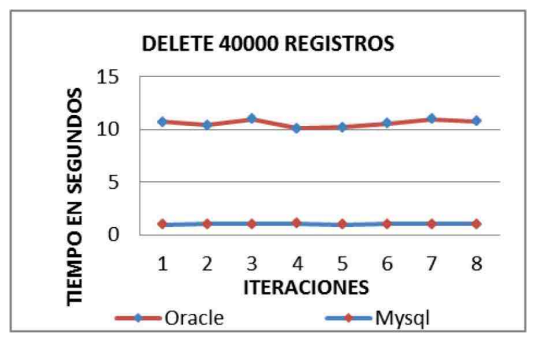

Fuente: elaboración propia.

Figura 17. Resultados prueba Delete 50000

Resultados obtenidos en tiempo por los dos motores de bases de datos al llevar a cabo el proceso de borrar 50000 registros

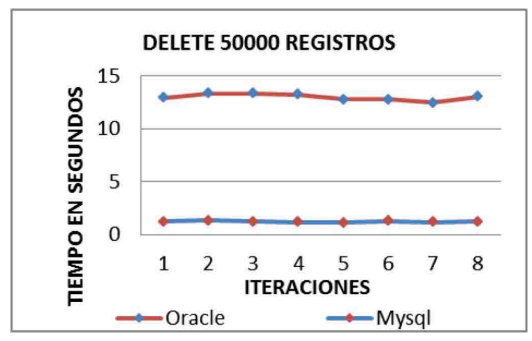

Fuente: elaboración propia.

De acuerdo con las Figuras 13, 14, 15, 16, y 17, se observa que el tiempo de ejecución de la sentencia DeLETE es muy extenso en el gestor Oracle, con un promedio total de 8,8 segundos en todo el proceso. En cambio, el gestor Mysql logra una mayor eficiencia, con un promedio total de 0,8 segundos en todo el proceso, es decir, que logra ejecutar la sentencia 9 veces más rápido.
Se observa asimismo que el gestor Mysql oscila entre los 0,3 y 1,3 segundos al ejecutar la inserción de los datos, mostrando así constancia en cada proceso. Por el contrario, Oracle muestra mucha variación, ya que sus tiempos de respuesta oscilan entre 4,2 y 13,3 segundos. Por cada 10000 registros borrados en el gestor Mysql, este se demora aproximadamente 0,19 segundos.

\section{RESULTADOS Y DISCUSIÓN}

A partir de las pruebas efectuadas y los resultados obtenidos (indicados en la Figura 18), se puede determinar que el sistema gestor Mysql obtuvo un mejor rendimiento en las sentencias de inserción (INSERT) y borrado (DELETE) de registros, demostrando más rapidez frente al gestor Oracle. Mysql es 1.8 veces, en promedio, más eficiente que Oracle. Los datos se presentan a continuación en las Figuras 18 y 19:

Tabla 12. Resultados finales pruebas Delete e Insert

\begin{tabular}{|c|c|c|c|c|c|}
\hline Sentencia $\rightarrow$ & Borr & ado & Inser & ción & \\
\hline & Tiempc & (seg) & Tiemp & (seg) & \\
\hline $\begin{array}{l}\text { Registros } \\
\text { Estudiados }\end{array}$ & $\begin{array}{l}\text { Promedio } \\
\text { Oracle }\end{array}$ & $\begin{array}{l}\text { Promedio } \\
\text { Mysql }\end{array}$ & $\begin{array}{l}\text { Promedio } \\
\text { Oracle }\end{array}$ & $\begin{array}{l}\text { Promedio } \\
\text { Mysql }\end{array}$ & $\begin{array}{l}\text { Mejor } \\
\text { Gestor }\end{array}$ \\
\hline 10000 & 5,439 & 0,431 & 79,999 & 28,494 & Mysql \\
\hline 20000 & 6,383 & 0,642 & 191,757 & 62,214 & Mysql \\
\hline 30000 & 8,877 & 0,875 & 270,604 & 82,863 & Mysql \\
\hline 40000 & 10,605 & 1,011 & 355,951 & 133,480 & Mysql \\
\hline 50000 & 13,005 & 1,216 & 415,732 & 153,169 & Mysql \\
\hline $\begin{array}{c}\text { PROMEDIO } \\
\text { TOTAL } \rightarrow\end{array}$ & 8,8618 & 0,835 & 262,808475 & 92,044 & Mysql \\
\hline
\end{tabular}

Fuente: elaboración propia.

Figura 18. Gráfico comparativo final sentencias Insert

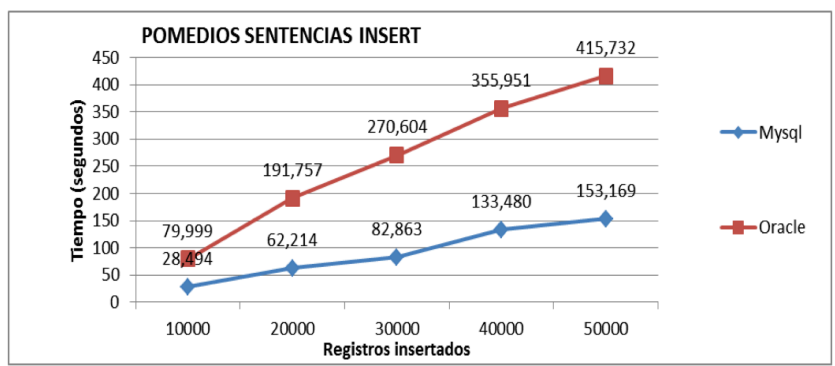

Fuente: elaboración propia. 
Figura 19. Gráfico comparativo final sentencias Delete

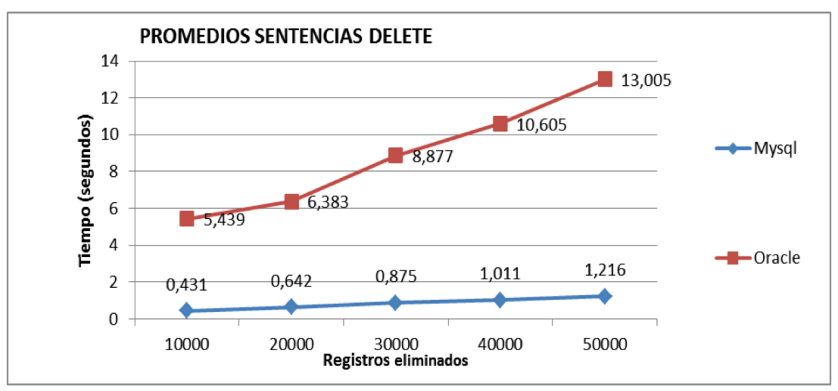

Fuente: elaboración propia.

De acuerdo con el análisis, se determina que en el contexto de las sentencias de consultas (SELECT), hay mayor eficiencia en Oracle con respecto a Mysql, como se muestra en la Tabla 13 y la Figura 20:

Tabla 13. Resultados finales sentencias Select

\begin{tabular}{|c|c|c|c|c|c|c|}
\hline \multirow[b]{2}{*}{$\begin{array}{r}\text { Grupo } \\
\text { No. }\end{array}$} & \multirow[b]{2}{*}{ Tipo de Agrupación } & \multirow[b]{2}{*}{ Consulta(s) } & \multirow[b]{2}{*}{$\begin{array}{l}\text { Registros } \\
\text { Obtenidos }\end{array}$} & \multicolumn{2}{|c|}{ Tiempo (seg) } & \multirow[b]{2}{*}{$\begin{array}{l}\text { Mejor } \\
\text { Gestor }\end{array}$} \\
\hline & & & & \begin{tabular}{|c|}
$\begin{array}{c}\text { Promedios } \\
\text { Oracle }\end{array}$ \\
\end{tabular} & $\begin{array}{c}\text { Promedios } \\
\text { Mysql }\end{array}$ & \\
\hline \multirow{2}{*}{1} & \multirow{2}{*}{ Consulta sencilla } & 1 & 50107 & 5,678 & \begin{tabular}{|l|}
1,807 \\
\end{tabular} & Mysal \\
\hline & & 2 & 50107 & 4,337 & 1,030 & Mysal \\
\hline 2 & Consulta única & 3 & 49106 & 0,896 & 0,002 & Mysal \\
\hline \multirow{2}{*}{3} & \multirow{2}{*}{ Condición múltiple } & 4 & 18561 & 1,310 & 1,041 & Mysal \\
\hline & & 5 & 47090 & 2,596 & 2,616 & Mysql \\
\hline 4 & Ordenación & 6 & 23335 & 4,130 & 9,821 & Oracle \\
\hline \multirow{2}{*}{5} & \multirow{2}{*}{$\begin{array}{l}\text { Agrupaciones y condiciones } \\
\text { de grupo }\end{array}$} & 7 & 19 & 1,249 & 0,082 & Mysal \\
\hline & & 8 & 9 & 1,146 & 0,071 & Mysal. \\
\hline 6 & $\begin{array}{l}\text { Combinación entre tablas } \\
\text { (JOIN SIMPLE) }\end{array}$ & 9 & 21053 & 0,907 & 0,982 & Oracle \\
\hline 7 & $\begin{array}{l}\text { Combinación entre tablas } \\
\text { (JOIN MÚLTIPLE) }\end{array}$ & 10 & 8018 & 0,991 & 1,328 & Oracle \\
\hline 8 & Subconsulta simple & 11 & 13842 & 6,982 & 10,463 & Oracle \\
\hline \multirow[t]{2}{*}{9} & Subconsulta múltiple & \begin{tabular}{l|l}
12 \\
\end{tabular} & 8781 & 8,439 & 10,077 & Oracle \\
\hline & & \multicolumn{2}{|c|}{ PROMEDIO TOTAL $\rightarrow$} & 3,222 & 3,277 & Oracle \\
\hline
\end{tabular}

Fuente: Consultas realizadas y resultados obtenidos en los dos motores de bases de datos con la sentencia Select (Díez del Valle \& Torreño, 2016).

Figura 20. Gráfico comparativo final sentencias select

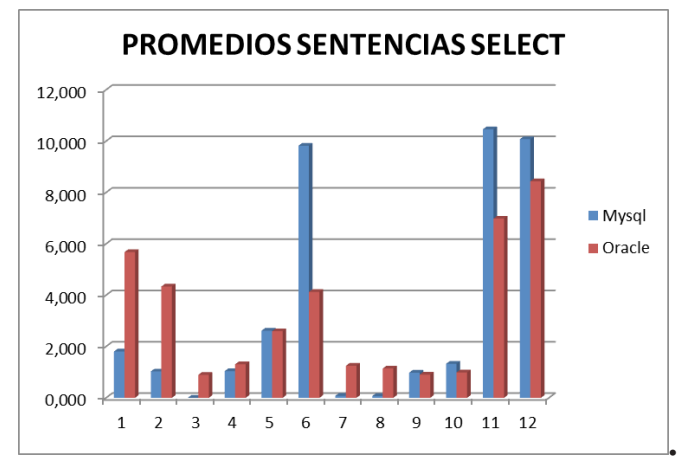

Fuente: elaboración propia.

Con el análisis realizado se determina que Oracle, a pesar de no haber demostrado buen funcionamiento en la mayoría de sentencias Select, logra superar a Mysql en el total de los resultados. Es de notar también que Oracle realiza más rápido las sentencias de consultas compuestas, mientras que Mysql lo hace solo en las sentencias de consultas básicas.

A continuación, en la Figura 21 se gráfica el rendimiento de Oracle, al cual le tomó menos tiempo, en promedio, que a Mysql para realizar las consultas. Sin embargo, su ventaja es solo de 0,05 segundos.

Figura 21. Gráfico comparativo promedio total sentencias Select

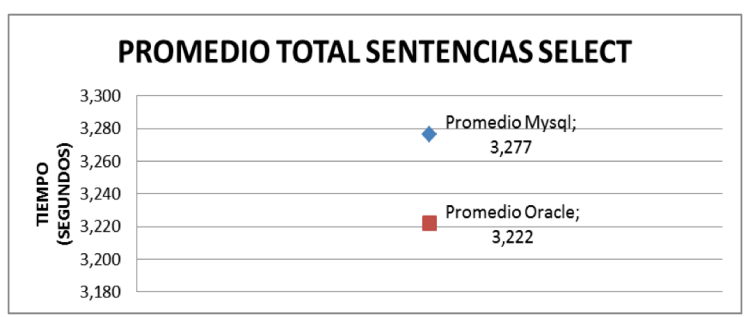

Fuente: Resultados finales del promedio de los dos motores de bases de datos aplicando las diferentes sentencias Select (Inzunza, 2016).

De acuerdo con la investigación realizada, se puede decir que el rendimiento en ambos motores de bases de datos es similar a la hora de realizar sentencias de consultas, pero no a la hora de insertar o borrar datos, ya que en este caso Mysql tiene ventaja frente a Oracle.

\section{CONCLUSIONES}

Este estudio ha logrado una revisión exhaustiva del rendimiento de dos Sistemas de Gestión de Bases de Datos Relacionales, Mysql y Oracle XE, que son ampliamente utilizados en el ámbito del aprendizaje informático y, además, en su versión de uso libre, ofrecen una amplia funcionalidad, incluyendo lo ejecutado en el presente análisis. Se obtiene un proceso comparativo real como resultado de las pruebas de las inserciones, consultas y eliminaciones de datos en cada uno de los gestores con comandos definidos en lenguaje SQL (Abad, 2015).

La evaluación de rendimiento permitió, entonces, mostrar de manera clara los datos estudiados, ya que, a su vez, facilitó agruparlos de manera congruente para la elección de un gestor de bases de datos acorde con la consulta que se vaya a realizar.

Se puede concluir que el gestor más funcional para quienes trabajan con una base de datos con 
demasiadas tablas y grandes cantidades de datos es Oracle, ya que a la hora de consultar registros que llevan a cabo muchos procesos tiene mejor rendimiento. Para el proceso de agregar datos y realizar pocas consultas, el más eficiente es utilizar Mysql. En últimas, Mysql es más rápido a la hora de insertar o borrar datos, mientras que Oracle es más rápido a la hora de consultar registros.

No obstante, cabe anotar que los análisis y resultados aquí descritos son usados para fines educativos. Esto, porque las características de los gestores descritos generalmente son las que se usan en un aula de clases o para aprendices, los cuales no cuentan con su versión completa.

\section{REFERENCIAS}

Abad, V. (2015). Estudio comparativo de BвD relacionales y No SQL en un entorno industrial [Tesis de doctorado]. Escuela Politécnica Nacional, Quito, Ecuador. Recuperado de https://riunet.upv.es/handle/10251/55530

Almonacid Inzunza, D. J. (2016). Comparación entre gestores de bases de datos relacionales [Tesis de doctorado]. Universidad Católica de la Santísima Concepción, Chile. Recuperado de http://repositoriodigital.ucsc.cl/handle/25022009/1092

Cardona, G. A., Rendón, F. N., \& Reyes, R. (2016). Prototipo para identificación de seguridad en envases de bebidas alcohólicas. Actas de Ingeniería, 2, 334-343. Recuperado de http:// fundacioniai.org/actas/Actas2/Actas2.38.pdf

Cuervo, M. C. (2012). Evaluación del rendimiento de los motores de bases de datos Mysql y Firebird. Revista Universidad EAFIT, 43(148), 78-90. Recuperado de http://publicaciones. eafit.edu.co/index.php/revista-universidad-eafit/article/view/703

Díez del Valle Medrano, Á. \& Torreño, F. (2016). Comparativa del rendimiento de consultas entre sistemas relacionales [Tesis de grado]. Universidad Politécnica, Madrid, España. Recuperado de http://oa.upm.es/42375/

García Frutos, R. (2017). Optimización de consultas en bases de datos relacionales [Tesis de grado]. Universidad Carlos III, Madrid, España. Recuperado de http: //hdl.handle.net/10016/24295
Gómez Ruiz, S. (2007). Microsoft sQL Server, Mysql y Postgre sQL. IPMagazine. Recuperado de https://www.protalia.com/articulos/52_53_54_55_56_57.pdf

HR Schema (2003). Oracle Database Sample Schemas. Recuperado de https://docs.oracle. com/cd/B13789_01/server.101/b10771/ scripts003.htm

López Herrera, P. (2016). Comparación del desempeño de los Sistemas Gestores de Bases de Datos Mysql y Postgre SQL [Tesis de grado]. Universidad Autónoma del Estado de México. Recuperado de http://ri.uaemex.mx/handle/20.500.11799/62548

Mysql. (2017). Límites en varias bases de datos y tablas. Recuperado de https: / /dev.Mysql.com/ $\mathrm{doc} / \mathrm{refman} / 5.5 / \mathrm{en} /$ database-count-limit. html

Oracle. (2017). Database Reference: Datatype Limits. Recuperado de http://docs.oracle. com/cd/B19306_01/server.102/b14237/limits001.htm\#i287903

Oracle Universit. (s. f). Oracle Database 12c: SQL Fundamentals. Recuperado de http://education.oracle.com/pls/web_prod-plq-dad/ db_pages.getpage?page_id=5001\&get_params=p_exam_id:1Z0061\#exam_preparation\&p_org_id=1000258\&lang=ESA

Pilco Guachi, L. (2012). Estudio Comparativo entre Sistemas de Gestion de Bases de Datos Libres FIREBIRD Y MYSQL en el Desarrollo de Aplicaciones Web [Tesis de grado]. Escuela Superior Politécnica de Chimborazo, Riobamba, Ecuador. Recuperado de http://dspace.espoch. edu.ec/handle/123456789/1534

Torres, L., López, F, \& Flores, P. (2016). Evaluador de sentencias de bases de datos en la formación de ingenieros. Anfei 5. Recuperado de http://www.anfei.org. $\mathrm{mx} /$ revista/index. $\mathrm{php} / \mathrm{revista/article/view/288/929}$

Zhingri, V., \& Augusto, C. (2016). Análisis de rendimiento entre la base de datos relacional: Mysql y una base de datos no relacional: MongoDB [Tesis de grado]. Universidad del Azuay, Cuenca, Ecuador. Recuperado de http: / / dspace.uazuay.edu.ec/handle/datos/5319 